\title{
Maternal microchimerism in the livers of patients with Biliary
} atresia

\author{
David L Suskind*, Philip Rosenthal ${ }^{1}$, Melvin B Heyman², Denice Kong 3 , \\ Greg Magrane ${ }^{1}$, Lee-Ann Baxter-Lowe ${ }^{2}$ and Marcus O Muench ${ }^{3}$
}

Address: ${ }^{1}$ Department of Pediatrics, University of California, San Francisco, USA, 2 Immunogenetics, University of California, San Francisco, USA and ${ }^{3}$ Laboratory Medicine, University of California, San Francisco, USA

Email: David L Suskind* - david.suskind@seattlechildrens.org; Philip Rosenthal - prosenth@itsa.ucsf.edu; Melvin B Heyman - mheyman@peds.ucsf.edu; Denice Kong - KongD@surgery.ucsf.edu; Greg Magrane - gmagrane@c.ucsf.edu; Lee-Ann BaxterLowe - baxterlowe@surgery.ucsf.edu; Marcus O Muench - muench@itsa.ucsf.edu

* Corresponding author

Published: 31 July 2004

BMC Gastroenterology 2004, 4:14 doi:10.1/86/1471-230X-4-14
Received: 06 May 2004

Accepted: 31 July 2004

This article is available from: http://www.biomedcentral.com/I47I-230X/4/14

(C) 2004 Suskind et al; licensee BioMed Central Ltd.

This is an open-access article distributed under the terms of the Creative Commons Attribution License (http://creativecommons.org/licenses/by/2.0), which permits unrestricted use, distribution, and reproduction in any medium, provided the original work is properly cited.

\begin{abstract}
Background: Biliary atresia (BA) is a neonatal cholestatic disease of unknown etiology. It is the leading cause of liver transplantation in children. Many similarities exist between BA and graft versus host disease suggesting engraftment of maternal cells during gestation could result in immune responses that lead to BA. The aim of this study was to determine the presence and extent of maternal microchimerism (MM) in the livers of infants with BA.
\end{abstract}

Methods: Using fluorescent in situ hybridization (FISH), II male BA \& 4 male neonatal hepatitis $(\mathrm{NH})$ livers, which served as controls, were analyzed for $X$ and $Y$-chromosomes. To further investigate $M M$ in BA, 3 patients with BA, and their mothers, were HLA typed. Using immunohistochemical stains, the BA livers were examined for MM. Four additional BA livers underwent analysis by polymerase chain reaction (PCR) for evidence of MM.

Results: By FISH, 8 BA and 2 NH livers were interpretable. Seven of eight BA specimens showed evidence of MM. The number of maternal cells ranged from 2-4 maternal cells per biopsy slide. Neither NH specimen showed evidence of MM. In addition, immunohistochemical stains confirmed evidence of MM. Using PCR, a range of I-I42 copies of maternal DNA per 25,000 copies of patients DNA was found.

Conclusions: Maternal microchimerism is present in the livers of patients with BA and may contribute to the pathogenesis of BA.

\section{Background}

Biliary atresia (BA) is a cholestatic disease of infancy characterized by the destruction of the biliary tree. [1-3] Both the intra-and extra-hepatic biliary ducts demonstrate evidence of a progressive destruction. This results in cholestasis, hepatic fibrosis and eventually cirrhosis. BA is associated with significant morbidity and mortality. Although the incidence of BA is 1 in 10,000 to 14,000 live births, [4,5] it accounts for over $40 \%$ of the neonatal cholestatic liver disease in Europe and the United States. [6] Prior to the hepatic portoenterostomy and liver transplantation, infants with BA had less than a $10 \%$ survival 
at 3 years of life and almost $100 \%$ mortality at 7 years of life. [7] In the United States, it is the leading cause of pediatric liver transplantation. [8] The etiology of biliary atresia remains unknown.

Many hypotheses on the etiology of BA exist. Two leading hypothesis are that BA occurs as a result of ductal plate malformations occurring during development of the liver, or as a result of an immune mediated process triggered by yet to be determined stimulus. [9] As more knowledge accrues about the histology and immunologic characteristics of $\mathrm{BA}$, the more it appears to be a progressive immune mediated process.

Graft versus host disease (GVHD), seen after allogeneic hematopoietic stem cell transplantation, is an immune mediated process triggered by the transfer of alloimmune cells from a donor to a host. GVHD shares many similarities with BA. Both BA and GVHD are characterized by a lymphocytic infiltration around the portal triad and damage to the biliary ducts. [10] The predominant lymphocytes in both disease are CD4+ $\mathrm{T}-$ helper (Th) 1 cells. [11-14] As with BA, in GVHD there is also an increase in cell adhesion molecules and human leukocyte antigen (HLA) class II markers. [11]

Given the similarities between BA and GVHD, we hypothesized that a contributing etiology of BA could be an alloimmune reaction such as in GVHD triggered by maternal microchimerism. Maternal microchimerism occurs when a small number of maternal cells are transferred to the offspring during pregnancy. This is known to occur in up to $40 \%$ of normal pregnancies. $[15,16]$ In addition, a clinical precedent of maternal microchimerism causing hepatic GVHD in children with severe combined immune deficiency (SCID) exists. [17] The aim of this study was to determine the presence and extent of maternal microchimerism in the livers of infants with BA.

\section{Methods}

This study was performed with the approval of the Committee of Human Research at USCF (H9048-20247-01). Cases of BA were identified through a data search in the Anatomic Pathology CoPath system by diagnosis or during patient medical visits to the UCSF Division of Pediatric Gastroenterology, Hepatology, and Nutrition. An explanation of the study was given to each study candidate. Written consent was obtained from each study participant.

\section{Fluorescent in situ hybridization (FISH) of male BA livers} We modified a previously reported method of FISH, [18] using $\mathrm{X}$ and Y-chromosomes probes. Y-chromosomes were stained with a green fluorescent dye, fluorescein isothiocyanate (FITC). X chromosomes were stained with a red fluorescent dye, cyanine 3 (Cy-3). Nuclear material was stained with a blue fluorescent dye 4", 6"-diamidino2-phenylindole (DAPI). After initial digestion and staining of the liver specimens, we examined the slides for evidence of female cells, depicted by two red signals, i.e. X chromosomes, with no green signal within the blue nuclear material. Slides were analyzed in a blinded fashion.

\section{HLA typing}

Patients with BA and their mothers were HLA typed. The HLA typing HLA-A, -B, and -DRB1 alleles of the child and mother were determined by sequence specific PCR (SSP) (Pel-Freez Clinical Systems, LLC ${ }^{\circledast}$, Brown Deer, WI, USA).

\section{Immunohistochemistry}

Frozen tissues sections of BA patient's livers $(5 \mu \mathrm{m})$ were fixed in acetone for 10 minutes at $4^{\circ} \mathrm{C}$ then washed in PBS ( 5 minutes centrifugation $\times 3$ ). Sections were blocked with Protein Block (Dako, Carpinteria, CA, USA) for thirty minutes at room temperature. Sections were then incubated with anti-HLA-B14 mouse monoclonal antibody (US Biological, Swampscott, Mass. USA) then washed in PBS. Sections were incubated in goat anti-mouse conjugated with FITC for 30 minutes at room temperature. Sections were rewashed and then counterstained with DAPI. Slides were then examined using a fluorescent microscope.

\section{Kinetic Polymerase Chain Reaction (kPCR)}

Using the HLA types of the patients with BA and their mothers, and a previously reported method of KPCR, evidence of maternal DNA was explored within the liver biopsy specimens of explanted BA livers. [18] The minor modifications to the kinetic PCR protocol included use of iCyler from BioRad (Hercules, Ca, USA) for amplification.

\section{Results}

\section{Detection of female cells in male BA liver biopsies}

Using FISH, we examined 11 male BA liver biopsies and 4 male NH liver biopsies. After initial digestion and staining of the liver specimens, we were able to interpret 8 of the BA and 2 NH liver biopsy specimens (Table 1). Three BA and $2 \mathrm{NH}$ biopsies underwent poor digestion and had no discernable signal. Figure 1 shows a liver from a male infant with BA. A male cell, depicted by a red signal, the $\mathrm{X}$ chromosome, and green signal, a Y chromosome within the blue nuclear material, clearly can be seen (A). In the same specimen, one can also see a female cell, depicted by two red signals, both $\mathrm{X}$-chromosomes with no green signal within the blue nuclear material (B). 7/8 BA specimens had evidence of maternal microchimerism. The number of maternal cells, per biopsy slide, was $2-4$ cells. Neither of the NH specimens had evidence of maternal microchimerism. 
Table I: Overview of maternal microchimerism in patients with BA and NH

\begin{tabular}{llll}
\hline Diagnosis & Chimerism & Method & $\begin{array}{l}\text { \# of Maternal cells/DNA per slide (FISH) or per 25,000 copies of } \\
\text { patient's DNA (kPCR) }\end{array}$ \\
\hline BA & Present & FISH & 2 maternal cells per slide \\
BA & Present & FISH & 2 maternal cells per slide \\
BA & Fresent & FISH & 4 maternal cells per slide \\
BA & Present & FISH+immunokistochemistry & 2 maternal cells per slide \\
BA & Present & FISH & -- \\
BA & Not interpretable & 2 maternal cells per slide \\
BA & Present & FISH & 3 maternal cells per slide \\
BA & Present & FISH & --- \\
BA & Not interpretable & FISH & -- \\
BA & Not interpretable & FISH & 0 maternal cells per slide \\
BA & None detected & FISH & 3 copies of maternal DNA per 25,000 copies of patient's DNA \\
BA & Present & kPCR & 1 copy of maternal DNA per 25,000 copies of patient's DNA \\
BA & Present & kPCR & 10 copies of maternal DNA per 25,000 copies of patient's DNA \\
BA & Present & kPCR & 142 copies of maternal DNA per 25,000 copies of patient's DNA \\
BA & Present & kPCR & 0 maternal cells per slide \\
NH & None detected & FISH & 0 maternal cells per slide \\
NH & None detected & FISH & --- \\
NH & Not interpretable & FISH & --- \\
NH & Not interpretable & FISH & \\
\hline
\end{tabular}

\section{Detection of maternal HLA antigen among BA liver samples}

To further investigate our findings, three patients with BA and their mothers were HLA typed. Using the HLA marker B14, we examined the BA livers for evidence of maternal microchimerism using immunohistochemistry techniques. An HLA-B14 positive patient served as our positive control (Figure 2). Specimen $\mathrm{B}$, our negative control, was a patient who was, along with his mother, negative for HLA-B14. Specimen C, our test specimen, was HLAB14-, with an HLA-B14 ${ }^{+}$mother. Specimen A (positive control) has a bright signal for HLA-B14 while there is only background staining for HLA-B14 in specimen B (negative control). Specimen C, the test specimen, has isolated scattered bright signals suggesting maternal microchimerism.

\section{Detection of maternal DNA by $k P C R$}

Finally, using polymerase chain reaction, in conjunction with the HLA typing of patients and mothers, we were able to affirm that maternal microchimerism occurs in the BA livers. An additional four fresh frozen liver specimens of BA infants, obtained at the time of transplantation, were examined for maternal DNA. All the livers had evidence of maternal microchimerism, with the range of 1 to 142 copies of maternal DNA detected per 25000 copies of patients' DNA. Figure 3 is a representative kPCR for maternal HLA-B40 in the second liver sample.
In all eleven of twelve patients with BA had evidence of maternal microchimerism by FISH or kinetic PCR, while neither of the two neonatal hepatitis patients had evidence of maternal microchimerism $(\mathrm{p}=0.03)$.

\section{Discussion}

$\mathrm{BA}$ is a progressive cholestatic disease of infancy, which is characterized by a mild to moderate lymphocytic infiltration within the extra and intrahepatic bile ducts. It is plausible, that maternal microchimerism may play a role in the etio-pathogenesis of BA by causing an alloimmune reaction given the similarities between $\mathrm{BA}$ and GVHD. We have shown that maternal microchimerism is present within the livers of patients with BA.

Maternal microchimerism occurs when maternal cells reside in the body of offspring. Maternal-fetal lymphocytic transfer is known to occur during pregnancy starting as early as the tenth week of gestation and continuing up to delivery. [19] The number of cells that traverse the placenta increases throughout this period. Schroder et al [15] demonstrated that approximately $1 / 10$ infants has about $0.07 \%$ maternal lymphocytes at the time of birth and more recent findings by Lo et al. [16] have indicated the presence of maternal cells in over $40 \%$ of fetal blood samples.

Disease has been shown to occur in mothers who have had fetal microchimerism, and children/adults who have had maternal microchimerism. Children who have 


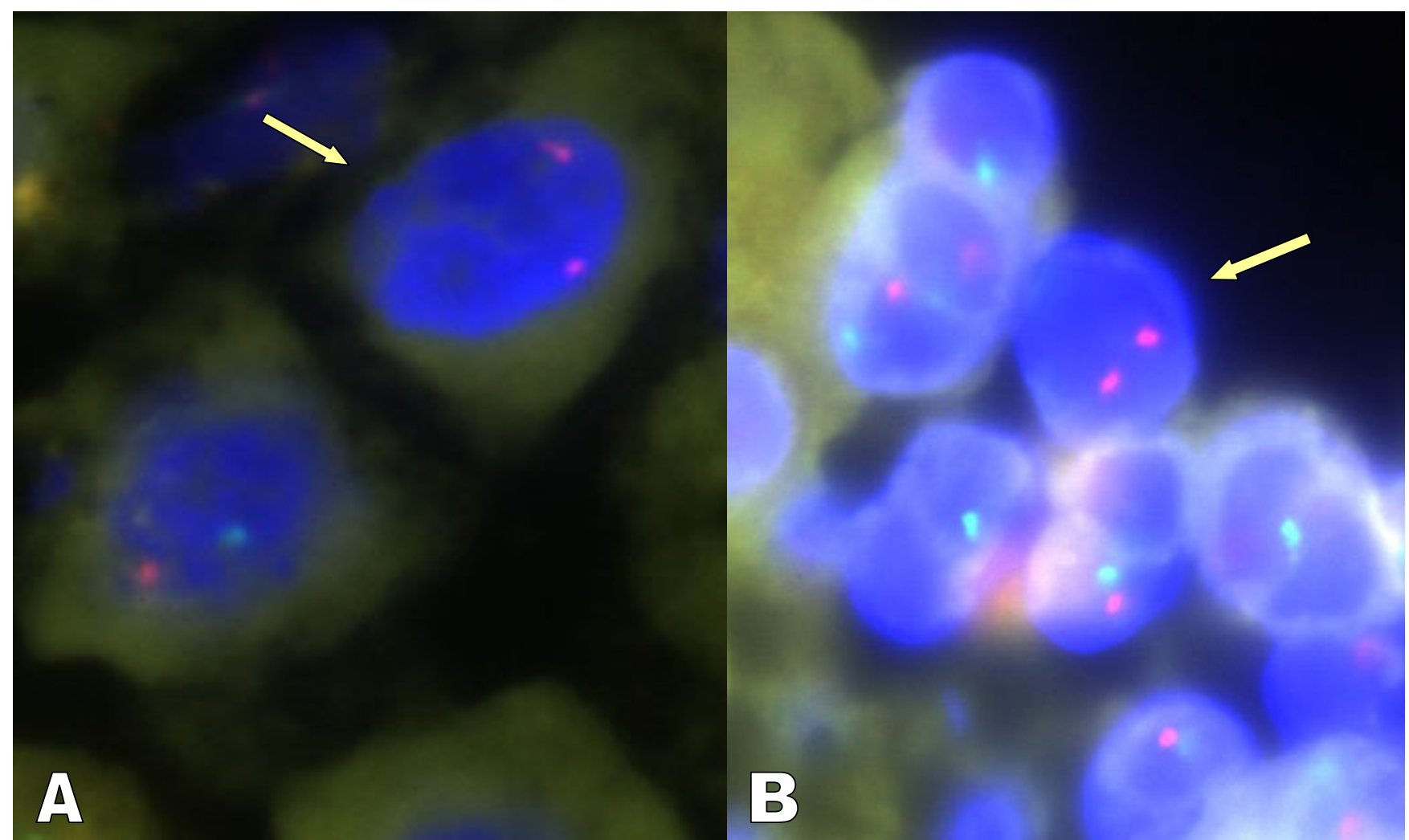

Figure I

A male cell, depicted by a red signal, the $X$ chromosome, and green signal, a $Y$ chromosome within the blue nuclear material, can be seen in both male BA liver specimens (A\&B). In the same specimen, one can also see a female cell, depicted by two red signals, both $\mathrm{X}$-chromosomes with no green signal within the blue nuclear material (arrow).

immunodeficiencies are at greater risk for graft verses host disease owing to engraftment of maternal lymphocytes. In both SCID and DiGeorge syndrome, maternal microchimerism and GVHD have been well described. In a study by Susanne Müller et al., 121 patients with SCID were evaluated for maternal microchimerism using HLA typing. Maternal cells were found in 48 patients, with 19 patients showing signs of GVHD. GVHD manifested itself in the skin and in the liver. [20] There have been numerous case reports showing GVHD in infancy of patients with DiGeorge. Manifestations of this disease include skin, gastrointestinal tract and liver involvement. [21]

The histologic and immunologic similarities of BA and GVHD are striking. The site of damage in BA and GVHD after bone marrow transplantation is the same. In each, lymphocytes congregate around the bile ducts. The damage occurs to both the intra-and extrahepatic biliary tracts. In a mouse model of acute GVHD, Nonomura et al. showed that transfer of allogeneic cells set along minor HLA mismatch can cause damage to both the intra and extrahepatic biliary ducts. Interestingly, the timeframe in this mouse model of acute GVHD, in terms of lymphocytic infiltration and fibrosis, appear similar to that of BA. [14] Initially, a peak of lymphocytes around the bile ducts occurs about 2 weeks after transfer of allogeneic cells from donor to host mouse; as the lymphocytic infiltration subsides, liver fibrosis increases. This correlates with disease progression in $\mathrm{BA}$, where initial diagnostic biopsies of BA livers usually show a larger lymphocytic infiltration, and less fibrosis, as compared to biopsies done later, i.e. at the time of liver transplantation. [22]

The lymphocytic infiltrates in both BA and GVHD are predominantly $\mathrm{CD}^{+} \mathrm{T}$ lymphocytes. The genes in BA livers showed differential lymphocytic function, with activation of osteopontin, a regulator of cell-mediated immunity in Th 1 cells, and interferon gamma. [23] Similarly, in GVHD, the predominant leukocyte is the CD4+ T lymphocyte. Although a variety of complex mechanisms are involved in causing the end organ damage in GVHD, evidence exists showing similarities in effector mechanisms. 


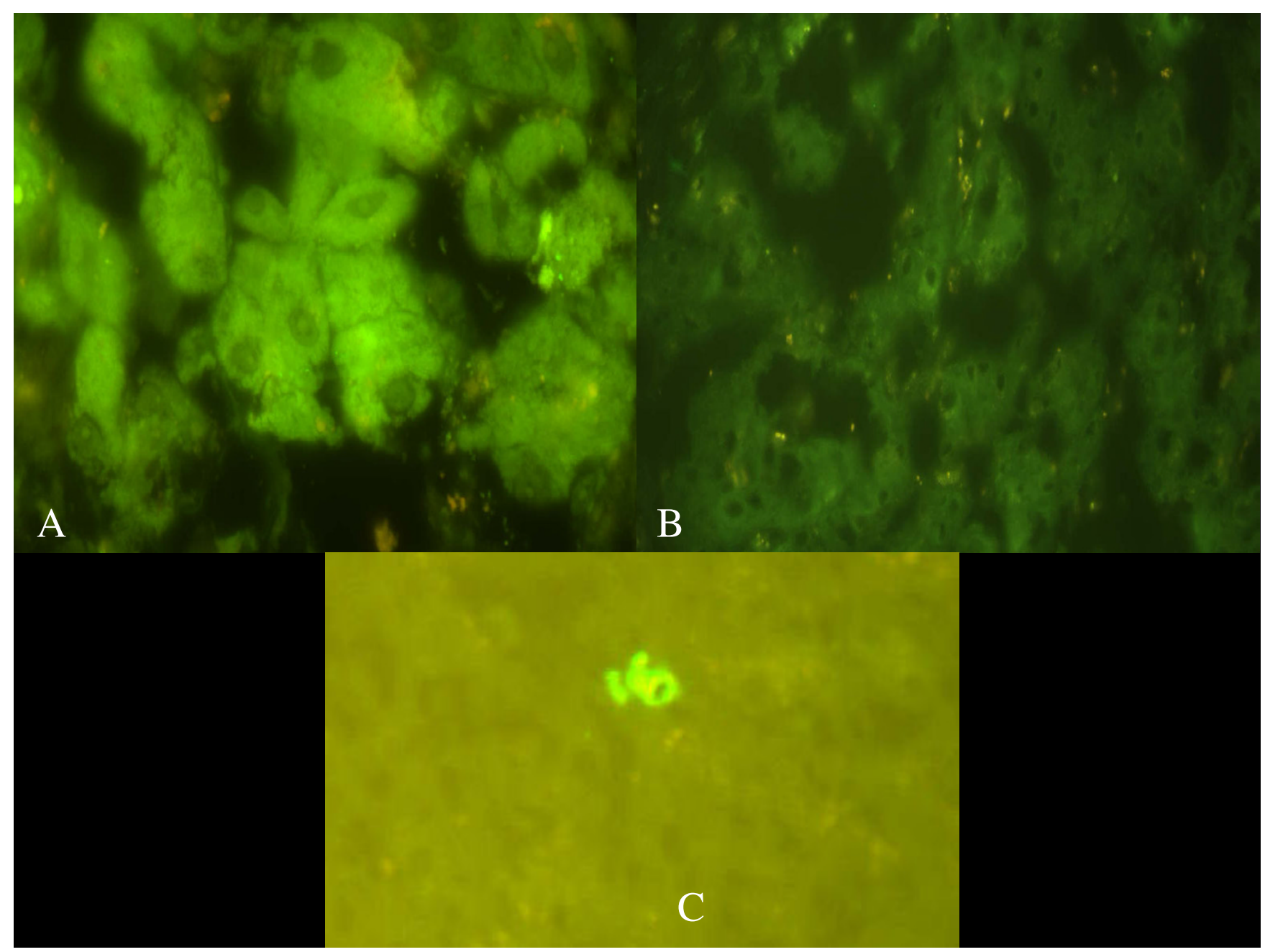

\section{Figure 2}

Using the HLA marker BI4, we examined the BA livers for evidence of maternal microchimerism using immunohistochemistry techniques. An HLA-BI4 positive patient served as our positive control (A). Specimen B, our negative control, was a patient who was, along with his mother, negative for HLA-BI4. Specimen C, our test specimen, was HLA-BI4 negative, with an HLABI4+ mother. Specimen A (positive control) has a bright signal for HLA-BI4 while there is only background staining for HLABI4 in specimen B (negative control). Specimen C, the test specimen, has isolated scattered bright signals suggesting maternal microchimerism.

In a SCID mouse model of acute GVHD, an increase in interferon-gamma secretion with a synchronized increase in activated Th cells was seen during acute GVHD. [24] The inflammatory responses in both BA and GVHD are also associated with increased expression of adhesion molecules such as CD54, and increased class II HLA markers, such as HLA-DR. HLA-DR and CD54, in both BA and GVHD, are seen predominantly around the bile ducts. $[11,25,26]$

Maternal microchimerism is not a rare occurrence in healthy individuals, but is usually not associated with dis- ease. The high occurrence of maternal microchimerism in healthy individuals has been suggested to have a tolergenic effect that may contribute to long-term microchimerism. [27]Additionally, in utero transplantation of haploidentical cells does not always lead to immune tolerance [28] and may lead to immune sensitization. Thus, the immunological consequences of the migration of maternal cells to the fetus appear to be variable. In most cases the maternal cells are likely to be cleared by the host's immune system or they may escape destruction by the immune system leading to engraftment. Engraftment of maternal immune cells in the fetal 

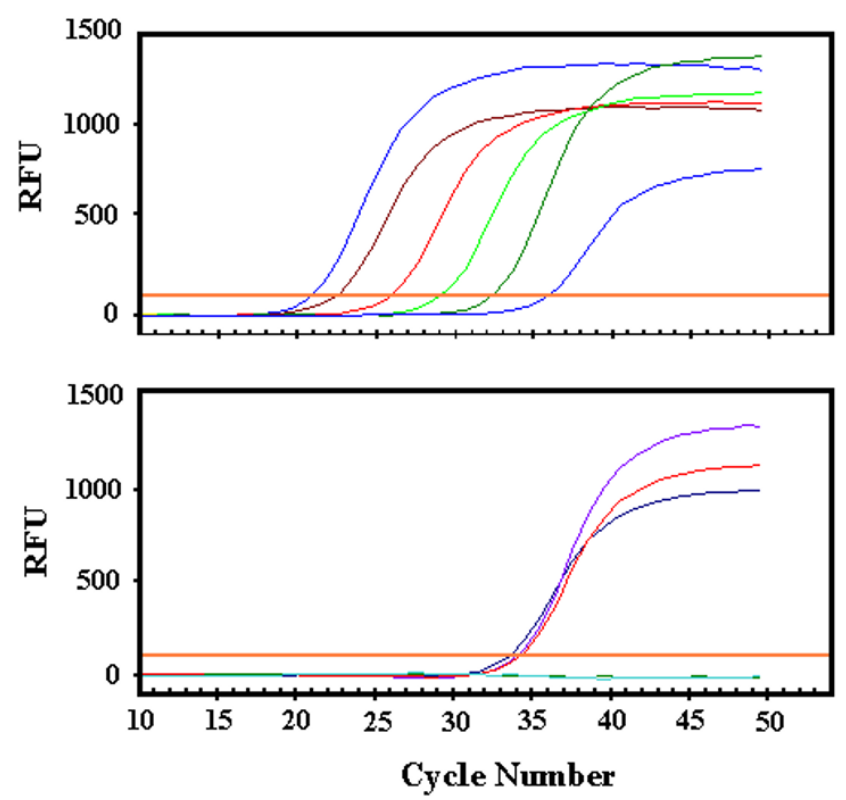

Figure 3

Representative kinetic PCR (kPCR) data for maternal HLAB40 in BA liver sample. SYBR Green fluorescence is plotted on the $y$-axis as a function of amplification cycle number on the $x$-axis. The upper panels depicts results for standard dilutions of HLA-B40 [left to right curves: 25000, 10000, 1000, $\mathrm{I00}, \mathrm{I0}$, and I genomic equivalence (gEq)]. A single copy of template produces fluorescent signal after approximately 36 cycles of amplification. The lower panel shows results from three liver replicates and two negative (no-template) controls. The three liver replicates have a positive signals at 2-5 $\mathrm{gEq}$, while the negative controls remained negative through 50 cycles.

biliary tract may result in an immune reaction against the host cholangiocytes. This is what we believe may occur in infants with BA. Alternatively, engrafted maternal cells in the fetus may subsequently be rejected by the immune system of the offspring, leading to destruction of the biliary tree.

Maternal microchimerism occurs in the livers of patients with BA. Given the previously well-described relationship between microchimerism and GVHD, and the similarities between BA and GVHD, these findings indicate a potential etio-pathogenisis for BA. Further investigations into the role of maternal microchimerism in BA are warranted.

\section{Competing interests}

None declared.

\section{Author's contributions}

DLS conceived of the hypothesis and contributed to the design and coordination of the study; he also drafted the manuscript. PR and MBH participated in the design of the study and manuscript preparation. DK carried out the kPCR and HLA typing analyses. GM performed the FISH analyses. LBL carried out the kPCR and HLA typing analyses and participated in the study design. MOM participated on the study design and coordination, immunohistochemistry stains and the manuscript preparation.

\section{Acknowledgements}

The authors wish to thank Drs. Yuet Wai Kan and Robert Suskind for their support and helpful suggestions. The invaluable support of Rebecca PH

Davis and Leslie Lewinter is also much appreciated.

Grant Support: This work was supported in part by NIH grants DK0606 17, 5 T32

DK07762 and DK5930I.

\section{References}

I. Hollander M, Schaffner F: Electron microscopic studies in biliary atresia. I. Bile ductular proliferation. Am J Dis Child 1968, I 1 6:49-56.

2. Hollander M, Schaffner F: Electron microscopic studies in biliary atresia. II. Hepatocellular alterations. Am J Dis Child 1968, I 16:57-65.

3. Sterling JA: Biliary tract morphology and prognosis of biliary atresia. Am J Gastroenterol 1966, 45:26I-266.

4. Fischler B, Haglund B, Hjern A: A population-based study on the incidence and possible pre- and perinatal etiologic risk factors of biliary atresia. Pediatr 2002, 141:2 17-222.

5. Ohi R, Ibrahim M: Biliary atresia. Semin Pediatr Surg 1992, I:II5-124.

6. Fischler B, Papadogiannakis N, Nemeth A: Aetiological factors in neonatal cholestasis. Acta Paediatr 200I, 90:88-92.

7. Karrer FM, Lilly JR, Stewart BA, Hall RJ: Biliary atresia registry, 1976 to 1989. J Pediatr Surg 1990, 25: 1076-80; discussion I08I.

8. Amersi F, Farmer DG, Busuttil RW: Fifteen-year experience with adult and pediatric liver transplantation at the University of California, Los Angeles. Clin Transpl 1998:255-26I.

9. Desmet VJ: Ludwig symposium on biliary disorders--part I. Pathogenesis of ductal plate abnormalities. Mayo Clin Proc 1998, 73:80-89.

10. Ben-Ezra JM, Stroup RM: Phenotype of bile ducts and infiltrating lymphocytes in graft-versus-host disease. Transplantation 1993, 56:162-165.

II. Broome U, Nemeth A, Hultcrantz R, Scheynius A: Different expression of HLA-DR and ICAM-I in livers from patients with biliary atresia and Byler's disease. J Hepatol 1997, 26:857-862

12. Chao NJ: Graft-versus-host disease: the viewpoint from the donor T cell. Biol Blood Marrow Transplant 1997, 3:1-10.

13. Ferrara JL, Levy R, Chao NJ: Pathophysiologic mechanisms of acute graft-vs.-host disease. Biol Blood Marrow Transplant 1999, 5:347-356.

14. Nonomura A, Kono N, Mizukami Y, Nakanuma Y: Histological changes of the liver in experimental graft-versus-host disease across minor histocompatibility barriers. VII. A light and electron microscopic study of the large bile duct. Liver 1993, 13:163-171.

15. Schroder J, Tiilikainen A, De la Chapelle A: Fetal leukocytes in the maternal circulation after delivery. I. Cytological aspects. Transplantation 1974, 17:346-354.

16. Lo YM, Lo ES, Watson N, Noakes L, Sargent IL, Thilaganathan B, Wainscoat JS: Two-way cell traffic between mother and fetus: biologic and clinical implications. Blood 1996, 88:4390-4395. 
17. Müller SM, Ege M, Pottharst A, Schulz AS, Schwarz K, Friedrich W: Transplacentally acquired maternal $T$ lymphocytes in severe combined immunodeficiency: a study of $12 \mathrm{I}$ patients. Blood 200I, 98:|847-I85|.

18. Reed W, Kong DZ, Lee TH, Cowan MJ, Busch MP, Baxter-Lowe LA: Non-invasive determination of the paternal HLA haplotype of a fetus using kinetic PCR to detect fetal microchimerism in maternal plasma. Bone Marrow Transplant 2002, 29:527-529.

19. Assche EL, Susanne C: Increase in the amount of fetal lymphocytes in maternal blood during pregnancy. J Med Genet 1980, 17:267-272.

20. Ocejo-Vinyals JG, Lozano MJ, Sanchez-Velasco P, Escribano de Diego J, Paz-Miguel JE, Leyva-Cobian F: An unusual concurrence of graft versus host disease caused by engraftment of maternal lymphocytes with DiGeorge anomaly. Arch Dis Child 2000, 83:165-169.

21. Ohya T, Fujimoto T, Shimomura H, Miyano T: Degeneration of intrahepatic bile duct with lymphocyte infiltration into biliary epithelial cells in biliary atresia. J Pediatr Surg 1995, 30:5 I5-518.

22. Bezerra JA, Tiao G, Ryckman FC, Alonso M, Sabla GE, Shneider B, Sokol RJ, Aronow BJ: Genetic induction of proinflammatory immunity in children with biliary atresia. Lancet 2002, 360:1653-1659.

23. Hu HZ, Li GL, Lim YK, Chan SH, Yap EH: Kinetics of interferongamma secretion and its regulatory factors in the early phase of acute graft-versus-host disease. Immunology 1999 , 98:379-385

24. Dillon PW, Belchis D, Minnick K, Tracy T: Differential expression of the major histocompatibility antigens and ICAM-I on bile duct epithelial cells in biliary atresia. Tohoku J Exp Med 1997, | 81:33-40.

25. Nakada M, Nakada K, Kawaguchi F, Wakisaka M, Kashimura T, Yamate N, Maeyama S, Uchikoshi T: Immunologic reaction and genetic factors in biliary atresia. Tohoku J Exp Med 1997 | 8 I:4|-47.

26. Shimazaki C, Ochiai N, Uchida R, Okano A, Fuchida S, Ashihara E, Inaba T, Fujita N, Maruya E, Nakagawa M: Non-T-cell-depleted HLA haploidentical stem cell transplantation in advanced hematologic malignancies based on the feto-maternal michrochimerism. Blood 2003, I 01:3334-3336.

27. Chen J., Chang M., Lee H., Muench M.O.: Haploidentical donor T cells fail to facilitate engraftment but lessen the immune response of host $\mathrm{T}$ cells in murine fetal transplantatio. British Journal of Haematology 2004, 1 26:277-384.

\section{Pre-publication history}

The pre-publication history for this paper can be accessed here:

http://www.biomedcentral.com/1471-230X/4/14/pre

pub
Publish with Bio Med Central and every scientist can read your work free of charge

"BioMed Central will be the most significant development for disseminating the results of biomedical research in our lifetime. "

Sir Paul Nurse, Cancer Research UK

Your research papers will be:

- available free of charge to the entire biomedical community

- peer reviewed and published immediately upon acceptance

- cited in PubMed and archived on PubMed Central

- yours - you keep the copyright

Submit your manuscript here:

http://www.biomedcentral.com/info/publishing_adv.asp
BioMedcentral 